\title{
The role of paroxysmal nocturnal hemoglobinuria in idiopathic habitual abortion
}

\author{
Yaren Dirik ${ }^{1}$, Ömer Ekinci ${ }^{1 *}$, Osman Kara ${ }^{2}$, Deniz Dirik ${ }^{3}$, Ali Doğan ${ }^{1}$, Cengiz Demir ${ }^{1}$ \\ ${ }^{1} V$ an Yuzuncu Yil University Faculty of Medicine, Department of Hematology, Van, Turkey \\ ${ }^{2} V$ an Education and Research Hospital, Department of Hematology, Van, Turkey \\ ${ }^{3} V$ an Education and Research Hospital, Department of Gynecology and Obstetrics, Van, Turkey
}

\begin{abstract}
Paroxysmal nocturnal hemoglobinuria $(\mathrm{PNH})$ which is an acquired clonal hematopoietic stem cell disease, usually presents with intravascular hemolysis, thrombosis and bone marrow failure. Hereditary and acquired thrombophilia are known to play significant role in the etiology of patients with habitual abortion (HA). PNH, which is a cause of acquired thrombophilia, may have a role in the etiology of HA. In the present study, we investigated the presence of PNH clone among the patients with a history of HA.

150 patients were enrolled in the study group, diagnosed with habitual abortus of unknown etiology and 150 healthy women with no history of habitual abortus as a control group. The age range for both groups was $18-55$ years. The PNH clone was screened by the FLAER (fluorescein-labeled proaerolysin) method.

The PNH clone was positive in five $(3.3 \%)$ patients in the study group. Four of the PNH clone positive patie nts were found to have a very low clone positivity level $(0.05 \%, 0.24 \%, 0.12 \%, 0.21 \%)$, while one had a high level $(30 \%)$.

PNH clone positivity results in the study group indicate that PNH should be investigated in cases of idiopathic HA, as one patient required treatment.
\end{abstract}

Key Words: Acquired thrombophilia, habitual abortion, paroxysmal nocturnal hemoglobinuria

\section{Introduction}

Paroxysmal nocturnal hemoglobinuria $(\mathrm{PNH})$ is an acquired clonal hemopoietic stem cell disease presenting with intravascular hemolysis, recurrent thrombosis and bone marrow failure. Mutations occur in the phosphatidylinositol glycan A (PIGA) gene linked to the $\mathrm{X}$ chromosome. A decrease in normal function of the glycerolphosphatidylinositol-anchor proteins (GPI-AP) encoded by this gene leaves the resulting blood cells vulnerable to complement attack, resulting in hemolysis, the main symptom of this disease (1). The prevalence of PNH is less than $1 / 200.000$ and the mean age of onset is approximately 42 , occurring in both sexes at the same frequency (2). Habitual abortion (HA) is considered the loss of pregnancy two or more times prior to the 20th week in the absence of mechanical or pharmacological intervention. HA is a condition that affects $1-3 \%$ of all women (3). Causes of HA include idiopathic causes (40-50\%), immunological factors (20-40\%), anatomical factors (10-15\%), endocrinological factors (10$15 \%$ ), genetic factors (5\%), infectious factors $(5 \%)$, and others (thrombotic factors, environmental factors, etc.; 10\%) (4).
Hereditary and acquired thrombophilia are known to play a vital role in the etiology of patients with a history of HA. Therefore, with regard to etiology, for hereditary thrombophilia genetic tests are performed, while for acquired thrombophilia, tests for systemic lupus erythematosus (SLE) and relevant diseases such as antiphospholipid antibody syndrome (AFAS) are conducted (5). However, in the etiology of HA, to date no studies have been carried out concerning $\mathrm{PNH}$, which is a cause of acquired thrombophilia. In present study, we aimed to investigate the existence of $\mathrm{PNH}$, a rare cause of thrombophilia, in the etiology of patients with a history of HA.

\section{Materials and Methods}

This study was performed between December 2015 and November 2016 at Van Yuzuncu Yil University in Van, Turkey with the patients who applied to the hematology department of the Faculty of Medicine. The HA histories of the patients were reviewed and 150 patients with no congenital causes were included in the study. These patients' ages ranged from 18 to 55 years. The control group consisted of 150 women aged 18-55 years with no history of habitual abortion who had been referred to the polyclinic for other 
Table 1. Descriptive statistics and comparison results for age, hemogram and biochemical results for the patient and control groups

\begin{tabular}{lccc}
\hline Parameter & $\begin{array}{c}\text { Number of patients } \\
(\text { Mean } \pm \text { SD })\end{array}$ & $\begin{array}{c}\text { Number of controls } \\
(\text { Mean } \pm \text { SD) }\end{array}$ & p value \\
\hline Age (years) & $28.97 \pm 6.45$ & $28.05 \pm 7.37$ & 0.274 \\
Hemoglobin (g/dL) & $13.34 \pm 1.56$ & $12.96 \pm 0.93$ & 0.01 \\
MCV (fL) & $84.34 \pm 7.04$ & $83.50 \pm 4.50$ & 0.221 \\
Total Leukocytes (x109/L) & $7357.82 \pm 1895.70$ & $7435.33 \pm 1514.01$ & 0.697 \\
Thrombocytes (x109/L) & $271.28 \pm 68.24$ & $275.89 \pm 71.51$ & 0.576 \\
Iron (ug/dL) & $73.90 \pm 32.17$ & $65.92 \pm 25.65$ & 0.02 \\
TIBC (ug/dL) & $376.04 \pm 63.31$ & $379.73 \pm 44.49$ & 0.560 \\
Ferritin (ng/mL) & $37.94 \pm 22.73$ & $27.00 \pm 16.72$ & 0.001 \\
Total Bilirubin (mg/dL) & $0.60 \pm 0.34$ & $0.82 \pm 0.26$ & 0.001 \\
LDH (IU/L) & $203.39 \pm 70.63$ & $189.27 \pm 24.61$ & 0.021 \\
\hline
\end{tabular}

reasons. The study was prospective, controlled and single-centered. Written informed consent was obtained from all volunteers who agreed to participate the study. Prior to the start of the study, approval was obtained from the Clinical Research Ethics Committee of the Faculty of Medicine of Van Yuzuncu Yil University.

Participants were questioned regarding age, pregnancy status, number of births and miscarriages, gestational age of habitual abortions, gynecological history, previous operations, presence of systemic disease (diabetes mellitus, hypertension, chronic liver or kidney disease, autoimmune diseases, etc.), and symptoms suggesting hemorrhagic diathesis or thrombosis, and whether the husband was a blood relative. The presence of PNH clone was examined by the fluorescence aerosol (FLAER) method. In addition, complete blood count (CBC) parameters (hemoglobin levels, white blood cell count, platelet count, mean corpuscular volume (MCV)), lactate dehydrogenase (LDH), total bilirubin, indirect bilirubin, iron, total iron binding capacity (TIBC) and ferritin levels were examined.

Statistical analysis: Descriptive statistics for studied variables (characteristics) were presented as mean and standard deviation. Student $\mathrm{t}$ test was performed to compare Control and Patient group. Statistical significance level was considered as $5 \%$ and SPSS (ver: 22) statistical program was used for all statistical computations.

\section{Results}

Epidemiological and laboratory characteristics for both study and control groups are shown in Table 1. The study groups' hemoglobin, iron, ferritin and $\mathrm{LDH}$ values were significantly higher than the control groups' $(\mathrm{p}=0.01, \mathrm{p}=0.02, \mathrm{p}=0.001$, $\mathrm{p}=0.02$, respectively). There wasn't a significant difference between the study group and the control group in terms of age, MCV, WBC, thrombocytes, and TIBC values $(p=0.27, p=0.22$, $\mathrm{p}=0.69, \mathrm{p}=0.57, \mathrm{p}=0.56$, respectively). Total bilirubin and indirect bilirubin levels were found significantly lower in the study group ( $\mathrm{p}=0.001$ for both).

Five women $(3.3 \%)$ in the patient group were tested positive for $\mathrm{PNH}$ clone using FLAER assay. The PNH clone was not detected in any women in the control group. The clone positivity rates in patients who tested positive were $30 \%, 0.05 \%, 0.24 \%, 0.12 \%$, and $0.21 \%$. No statistically significative difference was observed between $\mathrm{PNH}$ negative and $\mathrm{PNH}$ positive patients in the study group with respect to age, number of miscarriages, total leukocyte counts, hemoglobin, thrombocytes, ferritin, bilirubin levels, and LDH $(\mathrm{p}=0.956, \mathrm{p}=0.393, \mathrm{p}=0.719, \mathrm{p}=0.294$, $\mathrm{p}=0.316, \mathrm{p}=0.750, \mathrm{p}=0.426$, and $\mathrm{p}=0.128$, respectively).

The clone positivity of 4 of the PNH clone positive patients was very low titer without any remarkable clinical and laboratory findings and so they were followed-up by the hematology outpatient clinic without any treatment. A second FLAER assay was performed for one patient whose PNH clone titer was $30 \%$. The second test detected a PNH clone titer of $40 \%$, so the patient was diagnosed with PNH and Eculizumab treatment was started. The patient had had 3 miscarriages in a row and had never given birth to a live baby. The patient's medical history revealed that she had been diagnosed with and treated for iron deficiency on a number of occasions. The patient had no history of thrombosis. A direct antiglobulin (Coombs) test was found negative for hemolytic anemia. Results of the patient's laboratory analyses are shown in Table 2. 
Table 2. Laboratory results for patient with positive PNH clone at high titer

\begin{tabular}{lcc}
\hline Parameter & Result & Normal range \\
\hline Hemoglobin (g/dL) & 10.5 & $11-18$ \\
MCV (fL) & 85 & $80-100$ \\
Total Leukocytes (x109/L) & 5300 & $5000-10000$ \\
Thrombocytes (x109/L) & 55000 & $150000-400000$ \\
Iron (ug/dL) & 62 & $37-145$ \\
TIBC (ug/dL) & 316 & $112-346$ \\
Ferritin (ng/mL) & 30 & $14-150$ \\
Total Bilirubin (mg/dL) & 1.5 & $0.2-1.2$ \\
Indirect Bilirubin (mg/dL) & 1.3 & $0-0.8$ \\
LDH (IU/L) & 510 & $240-480$ \\
\hline
\end{tabular}

\section{Discussion}

Hemostatic precautions are taken to ensure blood fluidity on the fetomaternal surface in response to the predisposition towards coagulation during pregnancy. Trophoblastic invasion is performed to secure adequate fetal nutrition; however, the increase in thrombosis in these veins leads to different scenarios ranging from fetal growth retardation to intrauterine death. Thrombophilia is defined as susceptibility to thrombosis with changeable and unchangeable factors. These thrombophilic factors are believed to play a role in $55-62 \%$ of HA cases (6). It has been suggested that thrombophilic factors cause pregnancy loss by developing thrombosis in decidual veins (7). It is known that hereditary and acquired thrombophilia are known to play a vital role in the etiology of HA in patients with a history of HA. For this reason, the thrombophilic factors most commonly investigated with respect to etiology in HA patients are protein C and $\mathrm{S}$ deficiency, antithrombin III deficiency, factor $\mathrm{V}$ Leiden mutation, prothrombin G20210A gene mutation, MTHFR gene mutations, the presence of lupus anticoagulant, and anticardiolipin $\operatorname{IgM}$ and anticardiolipin $\operatorname{IgG}$ antibodies (8). There are a lot of studies performed on the relationship between $\mathrm{HA}$ and thrombophilia in the literature. Conflicting results have been found in studies investigating the relationship between MTHFR C677T and MTHFR A1298C mutations, which are accepted as uncertain risk factors for hypercoagulation and thrombophilia, and HA $(9,10)$. While some studies suggest that the presence of a mutation in a homozygous genotype or its presence together with other mutations increases the risk of HA, other studies report that these data do not indicate a risk for HA (11). A study by Dilley et al. (12) performed a study with 60 patients and 92 control subjects which showed that women who were carriers of factor $\mathrm{V}$, prothrombin, or the MTHFR mutation were at an increased risk for HA compared to women without these mutations. Foka et al., (13) in their study with 80 patients and 100 control subjects, proposed that factor $\mathrm{V}$ Leiden and prothrombin G20210A mutations may be risk factors for HA, but that MTHFR C677T homozygosis is not. The association between $\mathrm{HA}$ and antiphospholipid antibodies remains a focus of research on AFAS (14).

The mechanism and pathogenesis of developing thrombosis during the course of $\mathrm{PNH}$, which is a cause of acquired thrombophilia, have not yet been fully explained. However, thromboses that occur in the clinical course of the disease are among the primary causes of morbidity and mortality. Although thrombosis occurring in $\mathrm{PNH}$ patients can be observed in all types of vascular diseases, atypical and unexpected vascular thrombosis is more common. The development of thrombosis in the decidual veins has been put forth as a possible cause in HA etiology. We believe that thrombophilia occurring in the course of PNH may play a role in HA etiology by causing thrombosis in the decidual veins or by mechanisms not yet fully understood.

The correlation between PNH clone size and clinical symptoms of PNH has not yet been clearly defined. Although the course of thrombosis in $\mathrm{PNH}$ is not very clear, there are studies in the literature linking the risk of thrombosis to the size of the PNH clone (15). Two large studies have shown that in all patients with thrombosis, the PNH clone in granulocytes was greater than 50\% and $61 \%$ respectively $(15,16)$. However, in some studies, PNH clones have been reported at much lower levels in patients with thrombosis. Ageno et al. (17) in their study of 202 patients with splanchnic vein thrombosis reported low $\mathrm{PNH}$ clone levels $(0.014 \%$ and $0.16 \%)$ in two $(0.99 \%)$ patients, one with portal vein thrombosis without any risk factors and the other with superior mesenteric vein thrombosis due to inflammatory 
bowel disease. Therefore, the presence of thrombosis even with low PNH clones reported in the literature supports the suggestion that low levels of PNH clone positivity found in our study group may cause HA.

In conclusion; this is the first study to investigate the presence of $\mathrm{PNH}$, a cause of acquired thrombophilia in patients with HA. PNH should be considered in cases among which have a history of HA with an unknown etiology. In the presence of Coombs negative hemolytic anemia, bone marrow failure with cytopenia, atypical localized vascular thrombosis, and/or unexplained resistant iron deficiency in HA patients, the possibility of $\mathrm{PNH}$ is increased, and therefore PNH clone screening should be investigated.

\section{References}

1. Parker CJ. Paroxysmal Nocturnal Hemoglobinuria. In: Williams Hematology. 8th ed. Kaushansky K, ed. California, CA: McGrawHill; 2010: 521-531.

2. Hillmen P, Lewis SM, Bessler M, Luzzatto L, Dacie JV. Natural history of paroxysmal nocturnal hemoglobinuria. N Engl J Med 1995; 333: 1253-1258.

3. American College of Obstetricians and Gynecologists. Management of recurrent early pregnancy loss. ACOG Practice Bulletin 2001; 24: $1-8$.

4. Tabassum Parveiz MD. Recurrent Pregnancy Loss. JK-Practitioner 2003; 10: 323-326.

5. Preston FE, Rosendaal FR, Walker ID, et al. Increased fetal loss in women with heritable thrombophilia. Lancet 1996; 348: 913-916.

6. Rey E, Kahn SR, David M, Shrier I. Thrombophilic disorders and fetal loss: a metaanalysis. Lancet 2003; 361: 901-908.

7. Stevenson JL, Choi SH, Varki A. Differential metastasis inhibition by clinically relevant levels of heparins-correlation with selectin inhibition, not antithrombotic activity. Clin Cancer Res 2005; 11: 7003-7011.

8. Sood R. Thrombophilia and fetal loss: Lessons from gene targeting in mice. Thromb Res 2009; 123 Suppl 2: 79-84.

9. Kutteh WH, Park VM, Deitcher SR. Hypercoagulable state mutation analysis in white patients with early first- trimester recurrent pregnancy loss. Fertil Steril 1998; 71: 1048-1053.

10. Goodman CS, Coulam CB, Jeyendran RS, Vida AA, Roussev R. Which thrombophilic gene mutations are risk factors for recurrent pregnancy loss? Am J Rep Imnunol 2006; 56: 230-236.

11. Bick RL, Hoppensteadt D. Recurrent miscarriage syndrome and infertility due to blood coagulation protein/platelet defects: A review and update. Clin App Thromb Hemost 2005; 11: 1-13.

12. Dilley A, Benito C, Hooper WC, et al. Mutations in the factor $\mathrm{V}$, prothrombin and MTHFR genes are not risk factors for recurrent fetal loss. J Matern Fetal Neonatal Med 2002; 11: 176-182.

13. Foka ZJ, Lambropoulos AF, Saravelos H, et al. Factor V Leiden and prothrombin G20210A mutations, but not methylenetetrahydrofolate reductase $\mathrm{C} 677 \mathrm{~T}$, are associated with recurrent miscarriages. Hum Reprod 2000; 15: 458-462.

14. Rai RS, Regan L, Clifford K. Antiphospholipid antibodies and $\beta-2$ glycoprotein-I in 500 women with recurrent miscarriage: results of a comprehensive screening approach. Hum. Reprod 1995; 10: 2001-2005.

15. Nishimura J, Kanakura Y, Ware RE, et al. Clinical course and flow cytometric analysis of paroxysmal nocturnal hemoglobinuria in the United States and Japan. Medicine (Baltimore). Medicine 2004; 83: 193-207.

16. Moyo VM, Mukhina GL, Garrett ES, Brodsky RA. Natural history of paroxysmal nocturnal hemoglobinuria using modern diagnostic assays. Br J Haematol 2004; 126: 133-138.

17. Ageno W, Dentali F, De Stefano V, et al. Clonal populations of hematopoietic cells with paroxysmal nocturnal hemoglobinuria phenotype in patients with splanchnic vein thrombosis. Thromb Res 2014; 133(6): 1052-1055. 